\title{
Chemerin15-Ameliorated Cardiac
}

\section{Ischemia-Reperfusion Injury Is Associated with the Induction of Alternatively Activated Macrophages}

\author{
Chao Chang, ${ }^{1}$ Qingwei Ji, ${ }^{1}$ Bangwei Wu, ${ }^{2}$ Kunwu Yu, ${ }^{2}$ Qiutang Zeng, ${ }^{2}$ Shuanli Xin, ${ }^{3}$ \\ Jixiang Liu, ${ }^{3}$ and Yujie Zhou ${ }^{1}$ \\ ${ }^{1}$ Department of Cardiology, Beijing Anzhen Hospital, Capital Medical University, Beijing Institute of Heart, \\ Lung and Blood Vessel Disease, The Key Laboratory of Remodeling-Related Cardiovascular Disease, Ministry of Education, \\ Beijing 100029, China \\ ${ }^{2}$ Institute of Cardiovascular Diseases, Union Hospital, Tongji Medical College, Huazhong University of Science and Technology, \\ Wuhan 430022, China \\ ${ }^{3}$ Department of Cardiology, Handan First Hospital, Handan 056002, China
}

Correspondence should be addressed to Yujie Zhou; azzyj12@163.com

Received 20 April 2015; Revised 29 May 2015; Accepted 1 June 2015

Academic Editor: Ronald Gladue

Copyright (C) 2015 Chao Chang et al. This is an open access article distributed under the Creative Commons Attribution License, which permits unrestricted use, distribution, and reproduction in any medium, provided the original work is properly cited.

Chemerin15 (C15), an endogenous anti-inflammatory component, inhibits the activity of neutrophils and macrophages through $\mathrm{G}$ protein-coupled receptor ChemR23; however, its role as well as functional mechanism in mouse myocardial ischemia/reperfusion (I/R) injury remains unknown. Methods. Sham or I/R operations were performed on C57BL/6J mice. The I/R mice received an injection of $\mathrm{C} 15$ immediately before reperfusion. Serum troponin T levels, infarct size, cardiomyocyte apoptosis, reactive oxygen species (ROS) production, and infiltration of neutrophils were assessed $24 \mathrm{~h}$ after reperfusion, while the macrophage phenotypes, macrophage infiltration, and inflammatory cytokine levels were assessed $48 \mathrm{~h}$ after reperfusion. Results. Compared with the control group, the C15-treated mice showed an obvious amelioration of I/R injury and displayed less ROS, accompanied by reduced neutrophil recruitment. C15 decreased the tumor necrosis factor- (TNF-) $\alpha$ and interleukin- (IL-) 6 levels and increased the IL-10 levels in the serum of the I/R mice, which suggested a suppressed inflammatory response that could be related to elevated alternatively activated M2 macrophages with characteristic skewed expression of M2 markers and inhibition of classically activated M1 marker expression. Conclusion. C15 may induce alternatively activated M2 macrophage polarization and suppress the inflammatory response to protect against myocardial I/R injury in mice.

\section{Introduction}

Myocardial ischaemia/reperfusion (I/R) injury, an inflammatory condition, is characterized by the rapid influx of leukocytes, followed by cytokine secretion, increased oxidative stress, and protease release in the endangered myocardial region $[1,2]$. Prolonged ischemia and excessive inflammatory responses after $\mathrm{I} / \mathrm{R}$ are deleterious for cell survival and extracellular matrix integrity, resulting in irreversible cell apoptosis or necrosis, which further amplifies the inflammatory response and provokes a positive feedback loop to aggravate I/R injury $[1,3,4]$. Thus, interventions targeting leukocytes or inflammatory mediators could significantly ameliorate myocardial I/R injury $[2,5]$.

Improper macrophage activation is pathogenically related to various metabolic and immune-mediated inflammatory disorders, including myocardial I/R injury $[6,7]$. Macrophages express distinct patterns of surface receptors and metabolic enzymes in response to different stimuli, which ultimately results in diversified macrophage functions and phenotypes [8]. Broadly, two distinct polarization states of macrophages have been characterized, namely, M1 and M2 [9]. Classically activated M1 macrophages display a proinflammatory profile, whereas alternatively activated M2 
macrophages exhibit anti-inflammatory and tissue repair properties. M2 macrophages play a significant role in the immune system by preventing an excessive inflammatory response, which has been proven to be protective in various autoimmune diseases $[9,10]$. These M2 macrophages show modulation of the expression of cell surface receptors, leading to enhanced endocytic and phagocytic activities [11, 12]. Methods limiting M1 while promoting M2 polarization of macrophages could represent a unique therapeutic strategy to suppress inflammatory responses and ameliorate myocardial I/R injury [7, 13, 14].

Chemerin, a new member of the adipokine family, is highly expressed in placenta, liver, and white adipose tissues. To a lesser extent, chemerin is also expressed in many other tissues, such as the lungs, brown adipose tissue, heart, ovaries, kidneys, skeletal muscle, and pancreas. Acting through the receptor ChemR23, chemerin functions as a chemoattractant for neutrophils, macrophages, immature dendritic cells (DCs), and natural killer (NK) cells [15-18]. Circulating chemerin levels are elevated in numerous diseases associated with chronic inflammation, such as Crohn's disease, liver disease, psoriasis, arthritis, lupus, and atherosclerosis, which suggests that chemerin may play a proinflammatory role in autoimmune diseases [18-22]. Inactive prochemerin is converted to the bioactive chemerin isoform through proteolytic removal of the last 6 amino acids at its C-terminal by neutrophil-derived proteases (elastase and cathepsin $G$ ), mast cell products (tryptase), and proteases of the coagulation cascade $[23,24]$. One previous study demonstrated that the calpains and cathepsin $S$ derived from activated macrophages can cleave chemerin to generate the potent anti-inflammatory peptide chemerin15 (C15, A140-A154). C15 exhibits obvious anti-inflammatory properties, although its chemoattractant activity is reduced [25]. In a murine cutaneous excisional wound model, C15 promoted tissue repair and reduced scarring by dampening the immediate intravascular inflammatory response, including inhibition of leukocyte recruitment and macrophage activation [26]. In addition, a recent study showed that $\mathrm{C} 15$ reduced neutrophil recruitment and heart damage in a murine myocardial infarction model [27]; however, the mechanism for this effect has not been investigated.

In the present study, we hypothesized that $\mathrm{C} 15$ may induce M2 polarization, which would lead to further inhibition of the inflammatory response as well as decreased cardiomyocyte apoptosis and enhanced cardiac function after myocardial $\mathrm{I} / \mathrm{R}$.

\section{Materials and Methods}

2.1. Animals and Experimental Design. Male C57BL/6 mice (8-10 weeks of age) were purchased from the Beijing University (Beijing, China). The mice were fed a standard diet and given water ad libitum at the Animal Care Facility of Capital Medical University, according to the institutional guidelines. The mice underwent sham or I/R surgery. The present study included three experimental groups: sham, control I/R, and I/R + C15 (each group contained six to eight mice). The treatment group received C15 $(0.3 \mathrm{ng} / \mathrm{kg}$ dissolved in $200 \mu \mathrm{L}$ normal saline) (GL Biochem, China) through the tail vein before reperfusion [27]. In addition, C15 was repeatedly administered every $12 \mathrm{~h}$ over the $48 \mathrm{~h}$ reperfusion period. The serum troponin $\mathrm{T}$ levels and the infarct size were measured $24 \mathrm{~h}$ after reperfusion, and cardiac functions were assessed $48 \mathrm{~h}$ after reperfusion. Cell apoptosis, reactive oxygen species (ROS) production, and neutrophil infiltration were assessed $24 \mathrm{~h}$ after reperfusion, while macrophage phenotypes, macrophages infiltration, and inflammatory cytokine levels were measured $48 \mathrm{~h}$ after reperfusion [28]. All animal experiments were approved by the Animal Care and Utilization Committee of Capital Medical University, China.

\subsection{Myocardial I/R Injury In Vivo and Infarct Area Assessment.} The murine I/R model was performed as previously described [29]. The mice were anaesthetized by an intraperitoneal injection of pentobarbital sodium $(50 \mathrm{mg} / \mathrm{kg})$. The mice were orally intubated and then connected to a rodent ventilator. A left thoracotomy was performed through a horizontal incision at the third intercostal space. I/R was achieved by ligating the anterior descending branch of the left coronary artery with 8-0 silk suture, and silicon tubing (PE-10) was placed on top of the left anterior descending (LAD) coronary artery, $2 \mathrm{~mm}$ below the border between the left atrium and left ventricle (LV). Ischemia was confirmed by an electrocardiogram (ST elevation). The sham-operated animals were subjected to the same surgical procedures, except that the suture was passed under the LAD coronary artery but not tied. After $45 \mathrm{~min}$ of occlusion, the silicon tube was removed to achieve reperfusion, and the rib space and overlying muscles were closed. Echocardiographic analyses were performed before euthanasia. Twenty-four hours after reperfusion, the animals were reanesthetized, the heart function was blocked at the diastolic phase by administering potassium chloride $(20 \mathrm{mM})$ injection, the chest was opened, and the ascending aorta was cannulated and perfused with saline to wash out the blood. The LAD coronary artery was occluded with the same suture, which had been left at the site of ligation. To demarcate the ischemic area at risk (AAR), Alcian blue dye (1\%) was perfused into the aorta and coronary arteries. The hearts were excised, and the LVs were sliced into $1 \mathrm{~mm}$ thick cross sections. The heart sections were then incubated with a $1 \%$ triphenyltetrazolium chloride solution at $37^{\circ} \mathrm{C}$ for $15 \mathrm{~min}$. The infarct area (pale), AAR (not blue), and total LV area from both sides of each section were measured using ImagePro Plus software (Media Cybernetics, Inc., United Stated of America (USA)); the obtained values were averaged. The percentages of the area of infarction and AAR of each section were multiplied by the weight of the tissue section and then summed for all sections.

2.3. Serum Troponin T. The serum troponin $\mathrm{T}$ levels were measured as an index of cardiac cellular damage using a Quantitative Rapid Assay Kit (Roche Diagnostics GmbH, Germany). 
TABLE 1: Primer sequences for real-time PCR.

\begin{tabular}{lll}
\hline Gene & Forward $\left(5^{\prime}-3^{\prime}\right)$ & Reverse $\left(5^{\prime}-3^{\prime}\right)$ \\
\hline MR & ATGCCAAGTGGGAAAATCTG & TGTAGCAGTGGCCTGCATAG \\
Argl & CGCCTTTCTCAAAAGGACAG & ACAGACCGTGGGTTCTTCAC \\
iNOS & CACCTTGGAGTTCACCCAGT & ACCACTCGTACTTGGGATGC \\
COX-2 & GCGACATACTCAAGCAGGAGCA & AGTTGGTAACCGCTCAGGTGTTG \\
\hline
\end{tabular}

Argl, arginase 1; iNOS, inducible nitric oxide synthase; COX-2, cyclooxygenase-2.

2.4. Evaluation of Apoptosis in Tissue Sections. Deoxyribonucleic acid (DNA) fragmentation was detected in situ using terminal deoxynucleotidyl transferase-mediated dUTP nick end labeling (TUNEL), as previously described [30]. The excised hearts were fixed in $4 \%$ paraformaldehyde, embedded in paraffin, cut into $5 \mu \mathrm{m}$ thick sections, and treated as instructed in the In Situ Cell Death Detection Kit (Roche Diagnostics $\mathrm{GmbH}$, Germany). The cardiac caspase-3 activity was measured using a Caspase-3 Colorimetric Assay Kit, according to the manufacturer's instructions (Chemicon Pvt. Ltd., USA). The absorbance of 4-nitroaniline cleaved by caspase-3 was measured at $405 \mathrm{~nm}$ using a microplate reader (ELx800, BioTek Instruments, USA).

2.5. Lipid Peroxidation Detection. The ischemic zones were assessed for lipid peroxidation as an indicator of ROS activity. The supernatants of homogenized cardiac tissues were analyzed for lipid peroxidation products (malondialdehyde (MDA)/4-hydroxyalkenals) using a Lipid Peroxidation Assay Kit (Calbiochem, Germany).

2.6. Myeloperoxidase (MPO) Detection. Neutrophils infiltration was assessed with a Myeloperoxidase Activity Colorimetric Assay Kit (Biovision, USA) by measuring the MPO activity in the ischemic myocardium, according to the manufacturer's instructions.

2.7. Immunohistochemical Analysis. The cardiac tissues were fixed with $4 \%$ formalin solution, embedded in paraffin, and sectioned into $6 \mathrm{~mm}$ thick slices. To investigate macrophage infiltration after I/R injury, the sections were stained with the anti-mouse macrophage marker F4/80 (BD Biosciences, USA), following standard protocols.

2.8. Enzyme-Linked Immunosorbent Assay (ELISA) for Proinflammatory Factors. The serum levels of tumor necrosis factor- (TNF-) $\alpha$, interleukin- (IL-) 6 , and IL-10 were measured using a commercially available ELISA Kit (R\&D Systems, USA). All procedures were performed twice, and the results were reported in $\mathrm{pg} / \mathrm{mL}$.

2.9. Flow Cytometric Analysis. Forty-eight hours after reperfusion, the ischemic zones of cardiac tissues from I/R mice were obtained to analyze macrophage infiltration and phenotype using the following fluorochrome-labeled antibodies: allophycocyanin- (APC-) conjugated anti-mouse F4/80, phycoerythrin- (PE-) conjugated anti-mouse inducible nitric oxide synthase (iNOS), and PE-Cyanine7-conjugated antimouse CD206 (eBiosciences, USA).

2.10. Real-Time Polymerase Chain Reaction (PCR). Total ribonucleic acid (RNA) was extracted from the cardiac tissues using TRIzol reagent (Invitrogen, USA) and reversetranscribed into complementary DNA using the PrimeScript RT Reagent Kit (Takara Biotechnology, China), according to the manufacturer's instructions. The messenger RNA levels of the target genes were quantified using SYBR Green Master Mix (Takara Biotechnology), with the ABI PRISM 7900HT Sequence Detector System (Applied Biosystems, USA). Each reaction was performed twice, and the changes in the relative gene expression, which were normalized to glyceraldehyde 3phosphate dehydrogenase levels, were determined using the relative threshold cycle method. The primer sequences are shown in Table 1.

2.11. Statistical Analysis. The data were presented as the means \pm SEM. The differences between the two groups were evaluated using an unpaired Student's $t$-test. Oneway analysis of variance (ANOVA) was used for multiple comparisons, followed by Tukey's post hoc procedure for multiple range tests. All statistical analyses were performed using SPSS software, version 17.0 (SPSS, Inc., USA). A $P$ value less than 0.05 was considered statistically significant.

\section{Results}

3.1. C15 Alleviated Myocardial I/R Injury in Mice. The AAR : LV ratio did not differ significantly between the C15treated and untreated groups (Figure 1(a)), suggesting that the ligation was reproducibly performed on the same LAD level. In the I/R mice, the administration of $\mathrm{C} 15$ decreased the infarct size within the AAR by $35.2 \%$ compared to the untreated group (Figure 1(b)). Similarly, the serum level of cardiac troponin $\mathrm{T}$, a direct index of myocyte damage, was also significantly lower in the C15-treated group (Figure 1(c)). As shown in Figure 1(d) (by echocardiography), $48 \mathrm{~h}$ after $\mathrm{I} / \mathrm{R}$, the ejection fraction (EF) was significantly reduced in the I/R mice compared to the sham group. However, in the C15-treated group, high EF was observed at $48 \mathrm{~h}$ after reperfusion, indicating an obvious alleviation of I/R-induced cardiac dysfunction. These results showed that $\mathrm{C} 15$ could protect the heart against myocardial I/R injury in mice. 


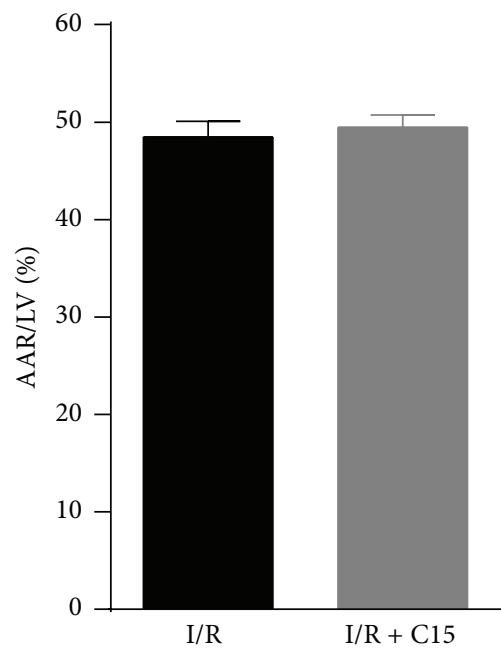

(a)

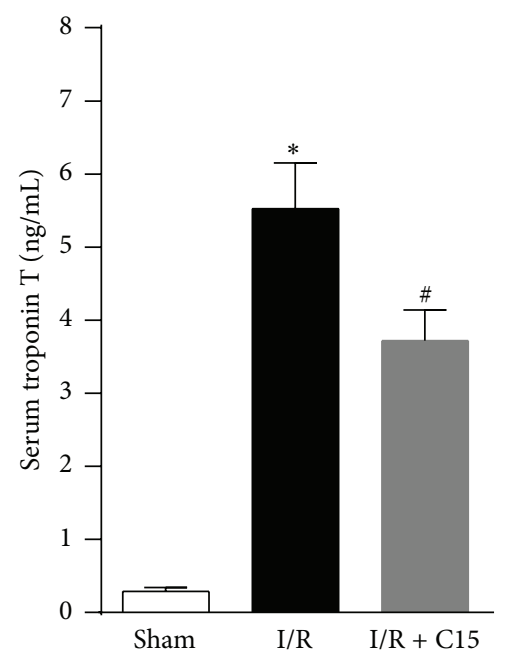

(c)

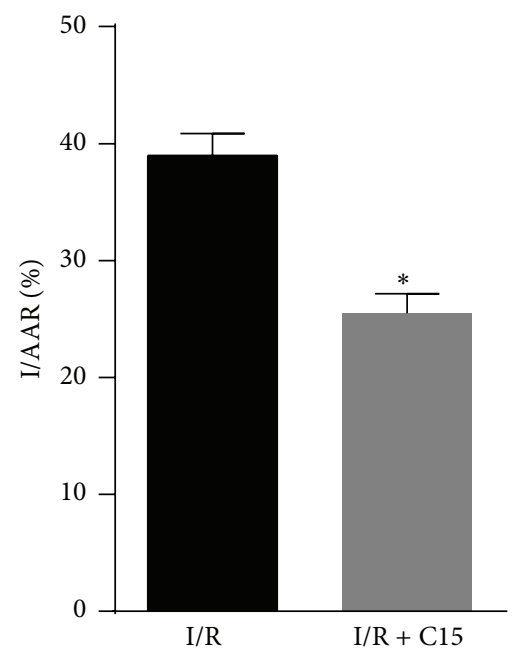

(b)

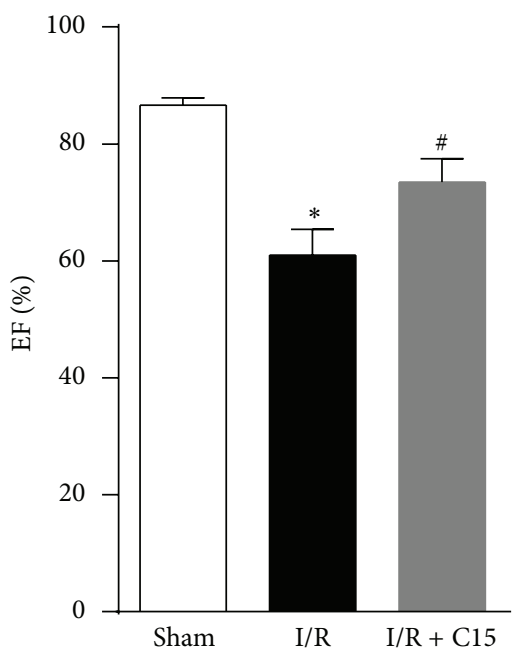

(d)

FIGURE 1: Chemerin15 (C15) decreased the myocardial infarct size and improved the hemodynamic performance following ischemia/reperfusion (I/R). ((a) and (b)) Quantification of the area at risk/left ventricular area (AAR/LV) and infarct area/AAR (I/AAR) $24 \mathrm{~h}$ after reperfusion $(n=6)$. (c) Serum cardiac troponin T $(\mathrm{cTnT})$ was measured in the sham, I/R, and I/R+C15 groups at $24 \mathrm{~h}$ after I/R $(n=5)$. (d) The ejection fraction $(\% \mathrm{EF})$ was measured $48 \mathrm{~h}$ after reperfusion $(n=6) . *<0.05$ versus the I/R or sham groups; \# $<0.05$ versus the I/R group.

3.2. C15 Reduced Cardiomyocyte Apoptosis and Neutrophil Infiltration. Cell apoptosis has been proposed to play an important role in myocardial I/R injury [31]. Compared with control mice, C15-treated I/R mice showed fewer apoptotic cells in the ischemic myocardium, as demonstrated by TUNEL staining (Figures 2(a) and 2(b)). Moreover, the C15 treatment significantly suppressed the activation of caspase3 in the ischemic myocardium of the I/R mice (Figure 2(c)). Furthermore, $\mathrm{C} 15$ treatment resulted in decreased neutrophil recruitment, as demonstrated by less MPO activity in the ischemic myocardium (Figure 2(e)). Moreover, the level of myocardial lipid peroxidation was significantly decreased in the C15-treated I/R mice compared with the control myocardium of I/R mice (Figure $2(\mathrm{~d})$ ). These results suggest that C15 may prevent cardiomyocyte apoptosis by inhibiting neutrophil activity.

3.3. C15 Generated Alternatively Activated Macrophages in the Ischemic Myocardium. Our results showed that C15 could significantly reduce macrophage infiltration in the ischemic myocardia of I/R mice (Figures 3(a) and 3(b)). To determine the effect of $\mathrm{C} 15$ treatment on macrophage polarization, the proportion of CD206 ${ }^{+}$cells among macrophages was measured. The C15-treated I/R mice displayed higher percentages of $\mathrm{CD} 206^{+} \mathrm{F} 4 / 80^{+} \mathrm{M} 2$ cells compared with the control group (Figure 3(d)), while the percentage of iNOS ${ }^{+} \mathrm{F} 4 / 80^{+} \mathrm{M} 1$ cells was decreased (Figure 3(c)). Furthermore, the C15 treatment increased the expression of M2 markers, including arginase 1 


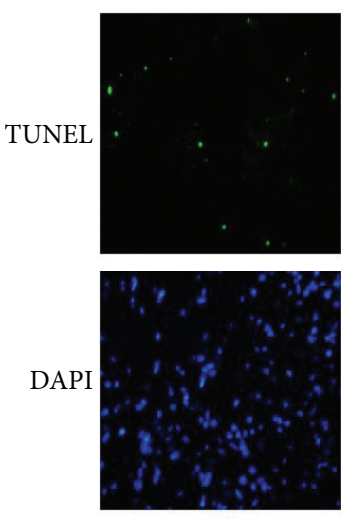

Sham
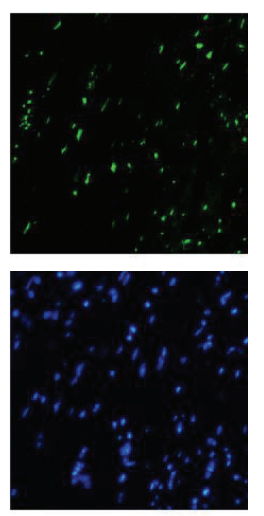

I/R
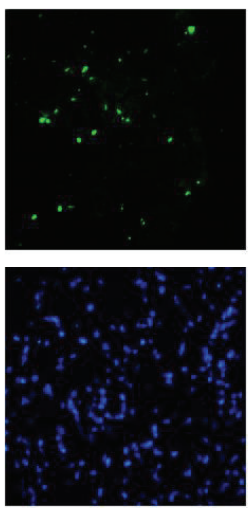

$\mathrm{I} / \mathrm{R}+\mathrm{C} 15$

(a)

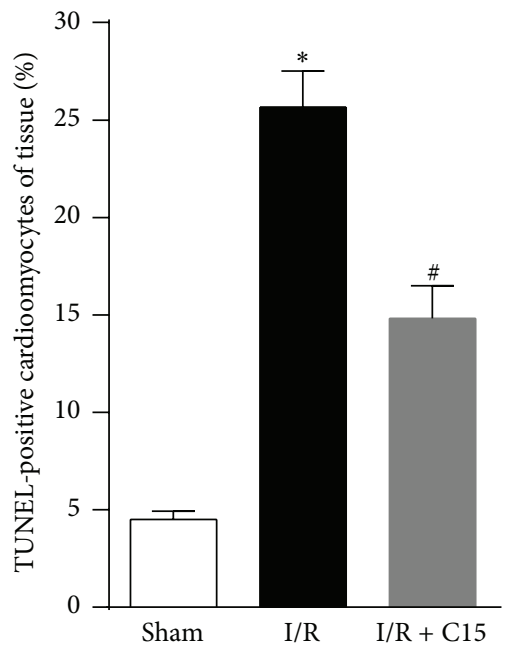

(b)

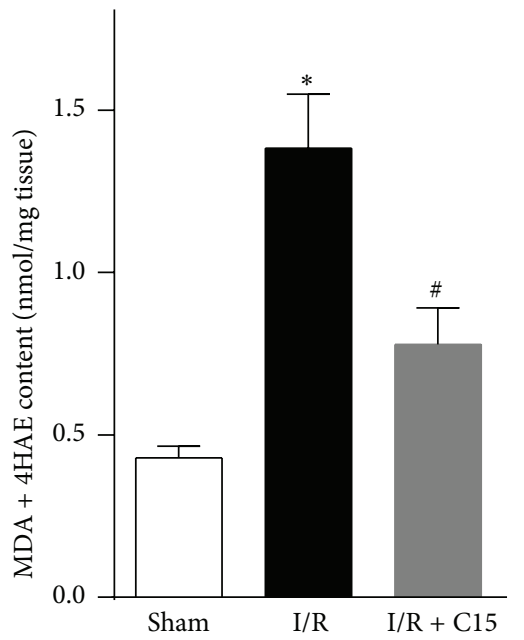

(d)

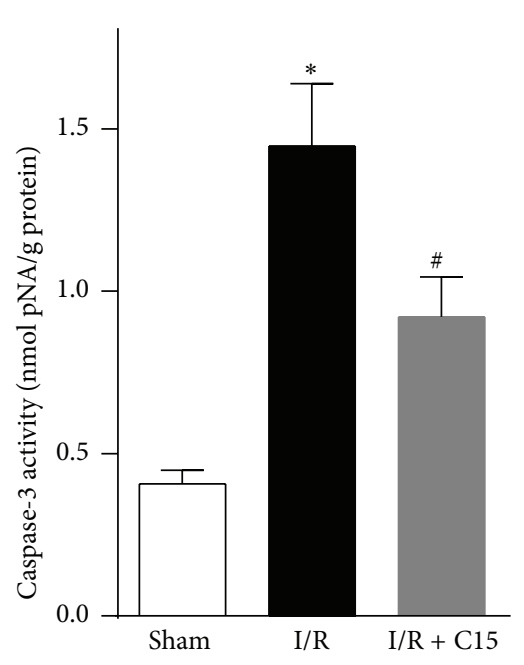

(c)

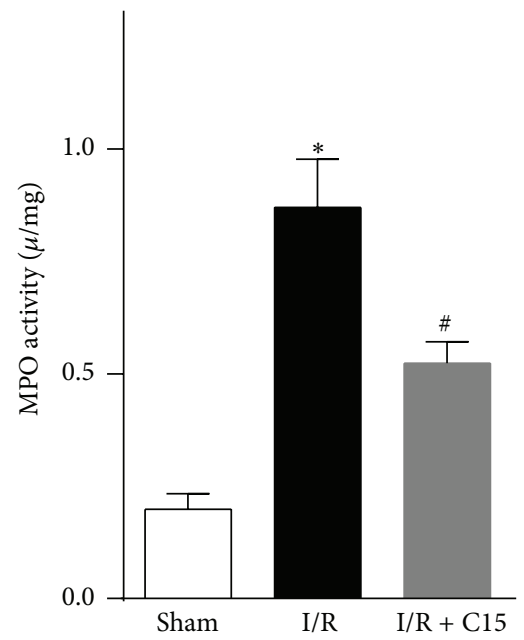

(e)

FiguRE 2: C15 reduced cardiomyocyte apoptosis and neutrophil infiltration. (a) Representative photographs of terminal deoxynucleotidyl transferase-mediated dUTP nick end labeling- (TUNEL-) stained ischemic area $24 \mathrm{~h}$ after reperfusion. For these images, apoptotic nuclei are indicated by TUNEL staining (green), and total nuclei are indicated by 4,6-diamidino-2-phenylindole (DAPI) staining (blue); bar, $10 \mu \mathrm{m}$. (b) Quantitative analysis of TUNEL-positive nuclei over the total number of nuclei in vivo $(n=6)$. (c) Caspase-3 activity in the ischemic myocardium was assessed after $24 \mathrm{~h}$ of reperfusion $(n=5)$. (d) Malonaldehyde+4-hydroxy-alkenals (MDA+4HAE) contents in the ischemic myocardium were evaluated using a lipid peroxidation assay $(n=5)$. (e) Cardiac myeloperoxidase (MPO) activity was detected in ischemic myocardia $(n=6) . * 0.05$ versus the sham group; \# 0.05 versus the I/R group. 

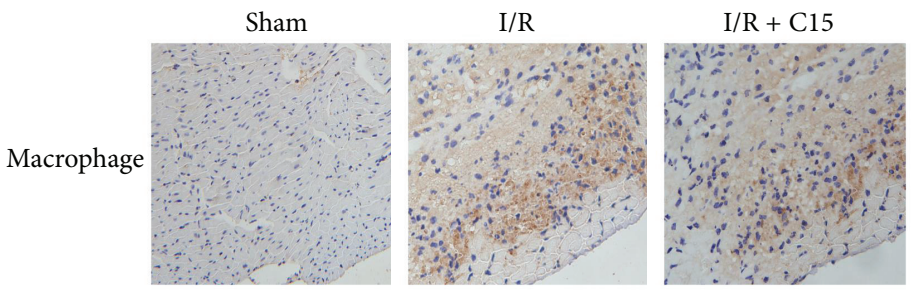

(a)

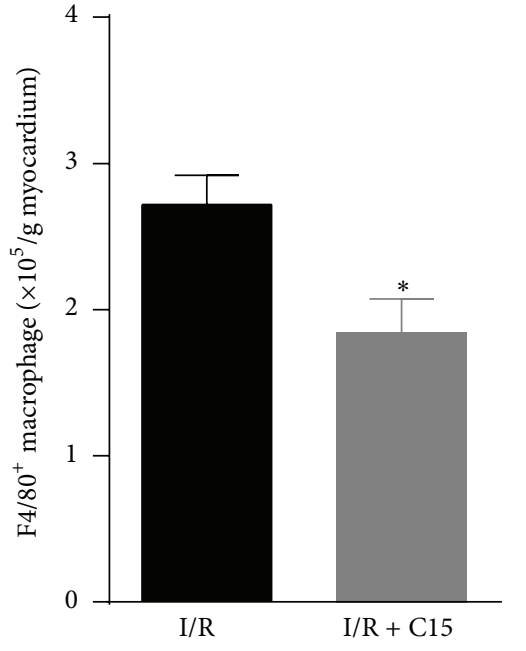

(b)

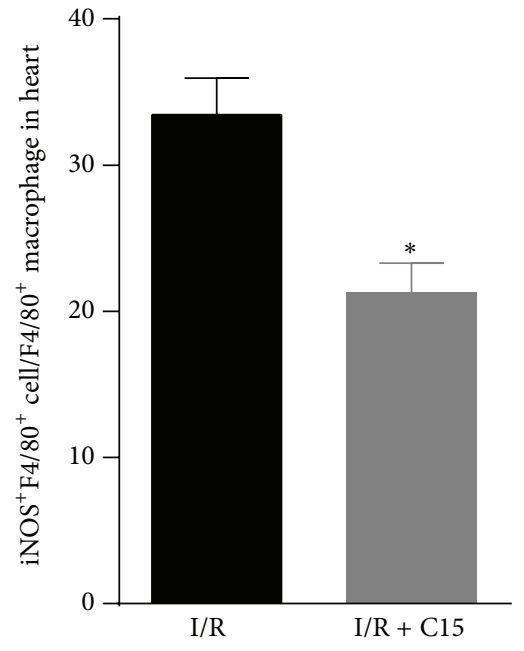

(c)

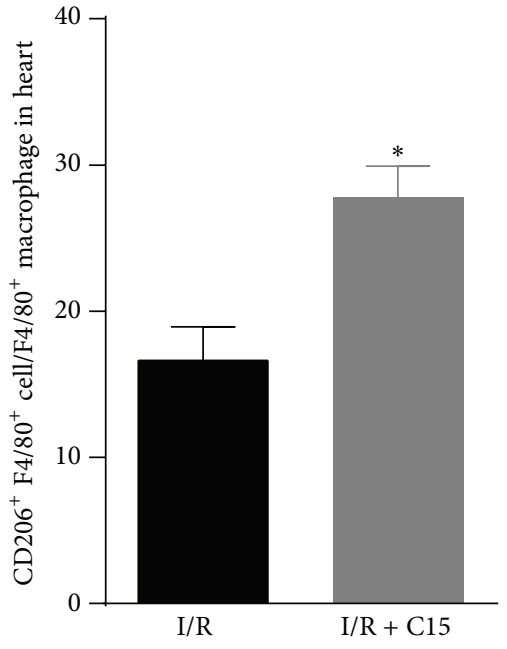

(d)

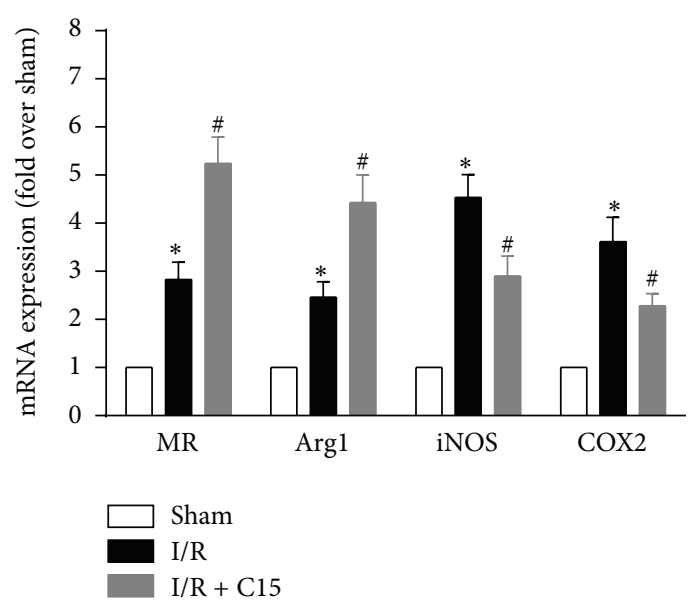

(e)

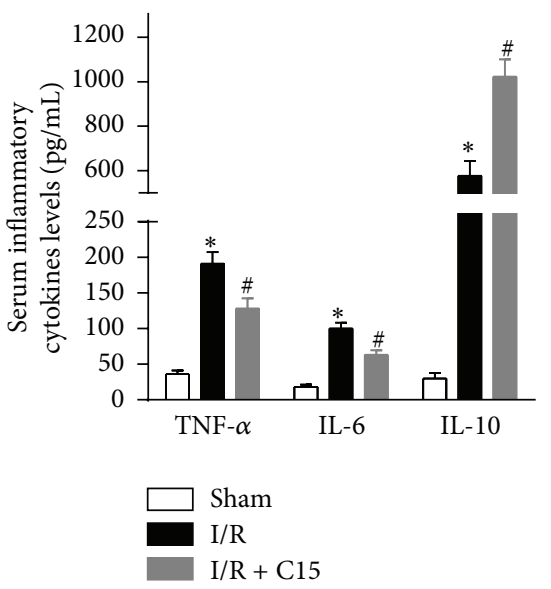

(f)

FIGURE 3: C15 generated alternatively activated macrophages in the ischemic myocardium. (a) Representative photographs of macrophage infiltration in the ischemic myocardium after $48 \mathrm{~h}$ of reperfusion. (b) The number of $\mathrm{F} 4 / 80^{+}$macrophages infiltrated into the ischemic myocardium was analyzed by flow cytometry $(n=6)$. (c) The number of classically activated M1 (iNOS ${ }^{+} \mathrm{F} 4 / 80^{+}$) macrophages infiltrated into the ischemic myocardium was analyzed with flow cytometry $(n=6)$. (d) The number of alternatively activated M2 $\left(\mathrm{CD} 206^{+} \mathrm{F} 4 / 80^{+}\right)$ macrophages that were infiltrated into the ischemic myocardium was analyzed with flow cytometry $(n=6)$. (e) M1 and M2 markers were analyzed with real-time PCR $(n=6)$. (f) The serum levels of proinflammatory cytokines (tumor necrosis factor- $\alpha($ TNF- $\alpha)$ and interleukin- 6 (IL-6)) and anti-inflammatory cytokines (IL-10) were measured after $48 \mathrm{~h}$ of reperfusion $(n=6) . *<0.05$ versus the I/R or sham groups; \#< 0.05 versus the I/R group.

(Arg1) and mannose receptor (MR), and inhibited the expression of M1 markers, including iNOS and cyclooxygenase-2 (COX-2) (Figure 3(e)), which was consistent with the flow cytometry results.
These results led us to hypothesize that the suppression of macrophage $\mathrm{M} 1$ activation by $\mathrm{C} 15$ may have led to changes in inflammatory cytokine expression. As shown in Figure 3(f), I/R increased the serum levels of TNF- $\alpha$ and IL-6, whereas 
C15 treatment resulted in significant suppression of the abovementioned proinflammatory cytokines. Furthermore, the level of IL-10 (an anti-inflammatory cytokine) was markedly increased in C15-treated I/R mice compared to control mice. Therefore, $\mathrm{C} 15$ may promote macrophage M2 polarization and thus maintain immune tolerance and confer protection against myocardial injury.

\section{Discussion}

The primary aim of the present study was to examine the role of $\mathrm{C} 15$ in mouse myocardial I/R injury and the protective mechanism of the actions involved in that process. Our results showed that $\mathrm{C} 15$ treatment clearly reduced the infarct size and improved cardiac function, and these results were accompanied by decreased cardiomyocyte apoptosis and neutrophil infiltration. Furthermore, $\mathrm{C} 15$ promoted alternative M2 macrophage activation and suppressed the proinflammatory response.

It is well known that a vigorous inflammatory response, mediated by several types of infiltrating leucocytes and related inflammatory cytokines, plays an important role in the pathogenesis of myocardial I/R injuries [1,2]. Recruited neutrophils and activated macrophages induce the expression of proinflammatory cytokines and increase both oxidative stress and protease release, which directly exacerbate the myocardial and endothelial injury $[3,7]$. Chemerin can stimulate pro- or anti-inflammatory responses, depending on the different pathophysiological conditions of various diseases [18]. Chemerin acts as a chemotactic factor for ChemR23expressing leukocyte populations, particularly macrophages, immature DCs, and NK cells, to promote their recruitment to localized sites of inflammation and tissue damage in conditions such as immunoglobulin E-mediated anaphylaxis, multiple sclerosis, and experimental autoimmune encephalomyelitis $[19,21,32]$. However, in mouse models of zymosan-induced peritonitis, lipopolysaccharide-induced acute lung inflammation, and excisional cutaneous skin wounds, the inhibition of endogenous chemerin activity or a loss of ChemR23 expression led to the exacerbation of inflammation and increased leukocyte infiltration, suggesting a protective role in these physiologic contexts $[16,25,26]$. Because neutrophils are typically the first cells to arrive at sites of inflammation [33], they can secrete serine proteases, predominantly cathepsin G, and elastase, which are responsible for the proteolytic cleavage of prochemerin to activated chem21-156 or chem21-157, respectively [24]. One previous study demonstrated that calpains and cathepsin $\mathrm{S}$ derived from activated macrophages could cleave chemerin to generate the potent anti-inflammatory peptide C15 (A140A154), which could then inhibit macrophage activation and inhibit their chemotactic activity [25]. Furthermore, $\mathrm{C} 15$ was shown to suppress the activation of integrins, which mediate neutrophil adhesion and transendothelial migration in vitro [27]. Direct administration of $\mathrm{C} 15$ restricted inflammatory cell recruitment to the wound site and improved wound repair in a mouse model of excisional cutaneous wounds [26]. In a zymosan-induced peritonitis model, intraperitoneal administration of C15 demonstrated a significant protective role, as shown by decreased neutrophil and monocyte recruitment as well as reduced proinflammatory cytokine expression [25]. Consistent with the findings of Cash et al. [27], the present study results also showed that C15 suppressed the infiltration of neutrophils and macrophages as well as inflammatory cytokine expression (TNF- $\alpha$, IL6), which may be related to decreased ROS production, cardiomyocyte apoptosis, and improved cardiac function in mouse myocardial I/R injuries [3, 31]. However, there was some disparity between reports in the extent to which the inflammatory response was inhibited and the protective effect mediated by $\mathrm{C} 15$. Nevertheless, these results suggest that the generation of $\mathrm{C} 15$ after proteolytic cleavage may function as a negative feedback mechanism to modulate an excessive inflammatory response by suppressing leucocyte infiltration and activation [25-27, 34]. In addition, C-terminal-truncated chemerin variants displayed greater chemotactic or antiinflammatory effects, which was the result with the cleavage at distinct sites by different classes of proteases [18, 23]. Thus, it could be speculated that the inconsistent role of chemerin between studies may derive from the existence of different classes of proteases in different disease conditions, which produce special levels of chemerin variants, leading to proor anti-inflammatory effects [18, 23-25].

The inflammatory response mediated by macrophages plays an important role in myocardial I/R injury [6,7]. In the present study, it was demonstrated that $\mathrm{C} 15$ could modulate macrophages toward an alternative M2 polarization state. In C15-treated myocardial I/R mice, the level of $\mathrm{F} 4 / 80^{+} \mathrm{CD} 206^{+}$ M2 macrophages in the ischemic myocardium was clearly higher than that in control I/R mice, which was accompanied by increased expression of M2 markers, including MR and Argl, whereas the expression of M1 markers, including iNOS and COX-2, was reduced. Macrophages exhibit heterogeneous functions, and their properties and activation state are modulated by local environmental stimuli $[9,11]$. Inadequate macrophage activation results in metabolic, inflammatory, and immune disorders that are pathogenically related to various autoimmune diseases [9, 10]. Proinflammatory cytokines (interferon- $\gamma, \mathrm{TNF}-\alpha$, and IL-6) induce macrophages towards "classically activated" M1 polarization, and these cells produce proinflammatory mediators, ROS, and proteases, resulting in damage to myocardial and endothelial cells in $\mathrm{I} / \mathrm{R}$ mice $[7,35]$. Conversely, $\mathrm{T}$ helper 2 cell-related cytokines, such as IL-4 or IL-13, can polarize macrophages towards an alternatively activated M2 phenotype associated with high levels of anti-inflammatory cytokines (IL-10, tumor growth factor- $\beta$ ) and the repair or remodeling of damaged tissues $[9,10]$. Cash et al. showed that C15 significantly enhanced macrophage phagocytosis of microbial particles and apoptotic cells in vitro and in vivo [34]. Apoptotic cell phagocytosis plays an important role in the resolution of inflammation and maintaining immune tolerance. Nevertheless, insufficient clearance of apoptotic cells leads to the accumulation of secondary necrotic cells, which can stimulate the inflammatory response because necrotic cell lysis releases cytotoxic and proinflammatory substances [36,37]. In autoimmune diseases, such as systemic 
lupus erythematosus, atherosclerosis, and diabetes mellitus, there is a persistent state of inflammation that may be related to the inefficient clearance of apoptotic cells and the resolution of inflammation $[38,39]$. Similar to other adipokines, adiponectin primes human monocyte differentiation into anti-inflammatory M2 macrophages through its ability to promote the clearance of apoptotic cells; during this process, adiponectin serves as a soluble bridge molecule between motifs on the surface of apoptotic cells and the phagocytic receptors on the surface of macrophages. Similarly, C15 may act in concert with prophagocytic signals, including Annexin A1 and Annexin-derived peptides, which are released by apoptotic cells to promote macrophage clearance [34, 40, 41]. In a mouse excisional cutaneous wound model, C15-treated mice expressed lower levels of TNF- $\alpha$ and iNOS (typically associated with an M1 phenotype) and higher levels of Arg1 (typically associated with an M2 phenotype) [26]. Furthermore, in C15-treated mice, macrophages appeared to have many protrusions mediated by actin cytoskeleton changes, which may be consistent with the report by McWhorter, who showed that modulation of macrophage shapes could affect macrophage polarization status [26, 42]. Collectively, these data indicate that $\mathrm{C} 15$ can skew macrophage towards alternative M2 polarization, thereby inhibiting the inflammatory response and leading to a protective role in heart damage after $\mathrm{I} / \mathrm{R}$ in a mouse model.

The mechanism through which $\mathrm{C} 15$ functions remains unclear. Earlier studies showed that C15 suppressed neutrophil migration and macrophage activation through a mechanism that was dependent on ChemR23 in vitro and in vivo $[25,27]$, which may seem to contradict the proinflammatory role of full-length chemerin. In addition to C15, ChemR23 has also been shown to be activated by lipid RvE1 to exert anti-inflammatory effects in animal models of zymosan-induced peritonitis and sulfonic acidinduced colitis [43-45]. Thus, ChemR23 may be a multifunctional receptor that transduces proinflammatory and antiinflammatory effects, and further investigation is needed to determine whether individual chemerin isoforms show differential bioactivity through one particular signaling pathway [18].

Together, the present study results show that C15 can reduce myocardial I/ $R$ injury in mice, as demonstrated by decreased cardiomyocyte apoptosis and improved heart function, which may be linked to suppressed proinflammatory responses mediated by alternative M2 macrophages. Thus, our results suggest that $\mathrm{C} 15$ may represent a new therapeutic class for the treatment of inflammatory diseases.

\section{Conflict of Interests}

The authors declare no competing interests.

\section{Acknowledgments}

This work was supported by the National Natural Science Foundation of China (Grand nos. 81160045 and 81270285), the Beijing Municipal High-Level Talent Foundation of
Health System (no. 2011-1-5), the Beijing Municipal Administration of Hospitals Clinical Medicine Development of Special Funding Support (Code: ZY201303), and the National Key Clinical Specialty Construction Project.

\section{References}

[1] N. G. Frangogiannis, C. W. Smith, and M. L. Entman, "The inflammatory response in myocardial infarction," Cardiovascular Research, vol. 53, no. 1, pp. 31-47, 2002.

[2] M. Y. Zuidema and C. Zhang, "Ischemia/reperfusion injury: the role of immune cells," World Journal of Cardiology, vol. 2, no. 10, pp. 325-332, 2010.

[3] K. A. Kaminski, T. A. Bonda, J. Korecki, and W. J. Musial, "Oxidative stress and neutrophil activation-the two keystones of ischemia/reperfusion injury," International Journal of Cardiology, vol. 86, no. 1, pp. 41-59, 2002.

[4] D. M. Yellon and D. J. Hausenloy, "Myocardial reperfusion injury," The New England Journal of Medicine, vol. 357, no. 11, pp. 1121-1135, 2007.

[5] S. Steffens, F. Montecucco, and F. Mach, "The inflammatory response as a target to reduce myocardial ischaemia and reperfusion injury," Thrombosis and Haemostasis, vol. 102, no. 2, pp. 240-247, 2009.

[6] J. M. Lambert, E. F. Lopez, and M. L. Lindsey, "Macrophage roles following myocardial infarction," International Journal of Cardiology, vol. 130, no. 2, pp. 147-158, 2008.

[7] L. Formigli, L. I. Manneschi, C. Nediani et al., "Are macrophages involved in early myocardial reperfusion injury?" Annals of Thoracic Surgery, vol. 71, no. 5, pp. 1596-1602, 2001.

[8] M. Nahrendorf and F. K. Swirski, "Monocyte and macrophage heterogeneity in the heart," Circulation Research, vol. 112, no. 12, pp. 1624-1633, 2013.

[9] A. Mantovani, A. Sica, and M. Locati, "Macrophage polarization comes of age," Immunity, vol. 23, no. 4, pp. 344-346, 2005.

[10] L. Cassetta, E. Cassol, and G. Poli, "Macrophage polarization in health and disease," TheScientificWorldJOURNAL, vol. 11, pp. 2391-2402, 2011.

[11] S. Gordon and F. O. Martinez, "Alternative activation of macrophages: mechanism and functions," Immunity, vol. 32, no. 5, pp. 593-604, 2010.

[12] F. Geissmann, S. Gordon, D. A. Hume, A. M. Mowat, and G. J. Randolph, "Unravelling mononuclear phagocyte heterogeneity," Nature Reviews Immunology, vol. 10, no. 6, pp. 453-460, 2010.

[13] J. A. Van Ginderachter, K. Movahedi, G. Hassanzadeh Ghassabeh et al., "Classical and alternative activation of mononuclear phagocytes: picking the best of both worlds for tumor promotion," Immunobiology, vol. 211, no. 6-8, pp. 487-501, 2006.

[14] V. Dayan, G. Yannarelli, F. Billia et al., "Mesenchymal stromal cells mediate a switch to alternatively activated monocytes/macrophages after acute myocardial infarction," Basic Research in Cardiology, vol. 106, no. 6, pp. 1299-1310, 2011.

[15] K. B. Goralski, T. C. McCarthy, E. A. Hanniman et al., "Chemerin, a novel adipokine that regulates adipogenesis and adipocyte metabolism," The Journal of Biological Chemistry, vol. 282, no. 38, pp. 28175-28188, 2007.

[16] S. Luangsay, V. Wittamer, B. Bondue et al., "Mouse ChemR23 is expressed in dendritic cell subsets and macrophages, and mediates an anti-inflammatory activity of chemerin in a lung 
disease model," Journal of Immunology, vol. 183, no. 10, pp. 6489-6499, 2009.

[17] S. Parolini, A. Santoro, E. Marcenaro et al., "The role of chemerin in the colocalization of $\mathrm{NK}$ and dendritic cell subsets into inflamed tissues," Blood, vol. 109, no. 9, pp. 3625-3632, 2007.

[18] J. L. Rourke, H. J. Dranse, and C. J. Sinal, “Towards an integrative approach to understanding the role of chemerin in human health and disease," Obesity Reviews, vol. 14, no. 3, pp. 245-262, 2013.

[19] K. L. Graham, B. A. Zabel, S. Loghavi et al., "Chemokinelike receptor-1 expression by central nervous system-infiltrating leukocytes and involvement in a model of autoimmune demyelinating disease," Journal of Immunology, vol. 183, no. 10, pp. 6717-6723, 2009.

[20] M. Kukla, K. Zwirska-Korczala, A. Gabriel et al., "Chemerin, vaspin and insulin resistance in chronic hepatitis C," Journal of Viral Hepatitis, vol. 17, no. 9, pp. 661-667, 2010.

[21] J. Tomalka-Kochanowska, B. Baranowska, E. Wolinska-Witort et al., "Plasma chemerin levels in patients with multiple sclerosis," Neuroendocrinology Letters, vol. 35, pp. 218-223, 2014.

[22] Q. Ji, Y. Lin, Z. Liang et al., "Chemerin is a novel biomarker of acute coronary syndrome but not of stable angina pectoris," Cardiovascular Diabetology, vol. 13, article 145, 2014.

[23] X.-Y. Du and L. L. K. Leung, "Proteolytic regulatory mechanism of chemerin bioactivity," Acta Biochimica et Biophysica Sinica, vol. 41, no. 12, pp. 973-979, 2009.

[24] V. Wittamer, B. Bondue, A. Guillabert, G. Vassart, M. Parmentier, and D. Communi, "Neutrophil-mediated maturation of chemerin: a link between innate and adaptive immunity," Journal of Immunology, vol. 175, no. 1, pp. 487-493, 2005.

[25] J. L. Cash, R. Hart, A. Russ et al., "Synthetic chemerin-derived peptides suppress inflammation through ChemR23," Journal of Experimental Medicine, vol. 205, no. 4, pp. 767-775, 2008.

[26] J. L. Cash, M. D. Bass, J. Campbell, M. Barnes, P. Kubes, and P. Martin, "Resolution mediator chemerin15 reprograms the wound microenvironment to promote repair and reduce scarring," Current Biology, vol. 24, no. 12, pp. 1406-1414, 2014.

[27] J. L. Cash, S. Bena, S. E. Headland, S. McArthur, V. Brancaleone, and M. Perretti, "Chemerin15 inhibits neutrophil-mediated vascular inflammation and myocardial ischemia-reperfusion injury through ChemR23," EMBO Reports, vol. 14, no. 11, pp. 999-1007, 2013.

[28] X. Yan, A. Anzai, Y. Katsumata et al., “Temporal dynamics of cardiac immune cell accumulation following acute myocardial infarction," Journal of Molecular and Cellular Cardiology, vol. 62, pp. 24-35, 2013.

[29] B. Wu, K. Meng, Q. Ji et al., "Interleukin-37 ameliorates myocardial ischaemia/reperfusion injury in mice," Clinical and Experimental Immunology, vol. 176, no. 3, pp. 438-451, 2014.

[30] S. Matsushima, J. Kuroda, T. Ago et al., "Broad suppression of NADPH oxidase activity exacerbates ischemia/reperfusion injury through inadvertent downregulation of hypoxia-inducible factor- $1 \alpha$ and upregulation of peroxisome proliferatoractivated receptor- $\alpha$," Circulation Research, vol. 112, no. 8, pp. 1135-1149, 2013.

[31] H. Fliss and D. Gattinger, "Apoptosis in ischemic and reperfused rat myocardium," Circulation Research, vol. 79, no. 5, pp. 949956, 1996.

[32] B. A. Zabel, S. Nakae, L. Zúñiga et al., "Mast cell-expressed orphan receptor CCRL2 binds chemerin and is required for optimal induction of IgE-mediated passive cutaneous anaphylaxis," The Journal of Experimental Medicine, vol. 205, no. 10, pp. 2207-2220, 2008.

[33] K. E. de Greef, D. K. Ysebaert, M. Ghielli et al., "Neutrophils and acute ischemia-reperfusion injury," Journal of Nephrology, vol. 11, no. 3, pp. 110-122, 1998.

[34] J. L. Cash, A. R. Christian, and D. R. Greaves, "Chemerin peptides promote phagocytosis in a ChemR23- and Syk-dependent manner," Journal of Immunology, vol. 184, no. 9, pp. 5315-5324, 2010.

[35] F. O. Martinez, A. Sica, A. Mantovani, and M. Locati, "Macrophage activation and polarization," Frontiers in Bioscience, vol. 13, no. 2, pp. 453-461, 2008.

[36] L. P. Erwig and P. M. Henson, "Immunological consequences of apoptotic cell phagocytosis," American Journal of Pathology, vol. 171, no. 1, pp. 2-8, 2007.

[37] R. E. Voll, M. Herrmann, E. A. Roth, C. Stach, J. R. Kalden, and I. Girkontaite, "Immunosuppressive effects of apoptotic cells," Nature, vol. 390, no. 6658, pp. 350-351, 1997.

[38] M. R. Elliott and K. S. Ravichandran, "Clearance of apoptotic cells: implications in health and disease," Journal of Cell Biology, vol. 189, no. 7, pp. 1059-1070, 2010.

[39] L. E. Mũoz, K. Lauber, M. Schiller, A. A. Manfredi, and M. Herrmann, "The role of defective clearance of apoptotic cells in systemic autoimmunity," Nature Reviews Rheumatology, vol. 6, no. 5, pp. 280-289, 2010.

[40] Y. Takemura, N. Ouchi, R. Shibata et al., "Adiponectin modulates inflammatory reactions via calreticulin receptordependent clearance of early apoptotic bodies," Journal of Clinical Investigation, vol. 117, no. 2, pp. 375-386, 2007.

[41] F. Lovren, Y. Pan, A. Quan et al., "Adiponectin primes human monocytes into alternative anti-inflammatory M2 macrophages," The American Journal of Physiology: Heart and Circulatory Physiology, vol. 299, no. 3, pp. H656-H663, 2010.

[42] F. Y. McWhorter, T. Wang, P. Nguyen, T. Chung, and W. F. Liu, "Modulation of macrophage phenotype by cell shape," Proceedings of the National Academy of Sciences of the United States of America, vol. 110, no. 43, pp. 17253-17258, 2013.

[43] M. Arita, F. Bianchini, J. Aliberti et al., "Stereochemical assignment, antiinflammatory properties, and receptor for the omega3 lipid mediator resolvin E1," Journal of Experimental Medicine, vol. 201, no. 5, pp. 713-722, 2005.

[44] M. Arita, T. Ohira, Y. P. Sun, S. Elangovan, N. Chiang, and C. N. Serhan, "Resolvin E1 selectively interacts with leukotriene B4 receptor BLT1 and ChemR23 to regulate inflammation," Journal of Immunology, vol. 178, no. 6, pp. 3912-3917, 2007.

[45] M. Arita, M. Yoshida, S. Hong et al., "Resolvin E1, an endogenous lipid mediator derived from omega-3 eicosapentaenoic acid, protects against 2,4,6-trinitrobenzene sulfonic acidinduced colitis," Proceedings of the National Academy of Sciences of the United States of America, vol. 102, no. 21, pp. 7671-7676, 2005. 


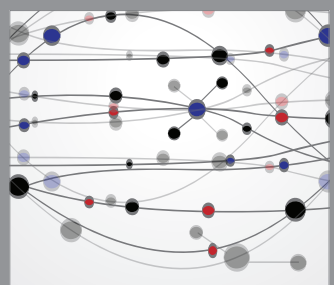

The Scientific World Journal
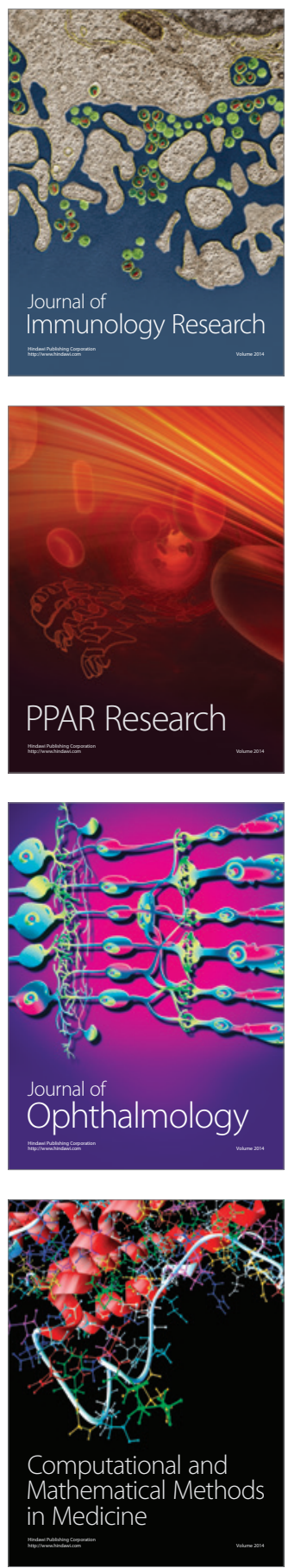

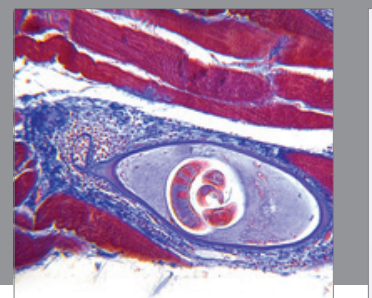

Gastroenterology

Research and Practice
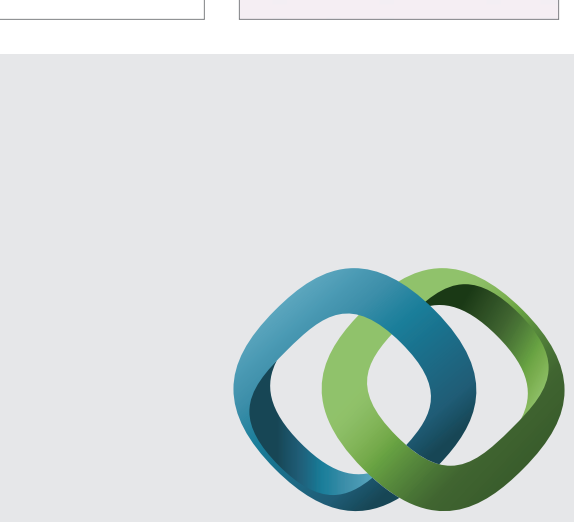

\section{Hindawi}

Submit your manuscripts at

http://www.hindawi.com
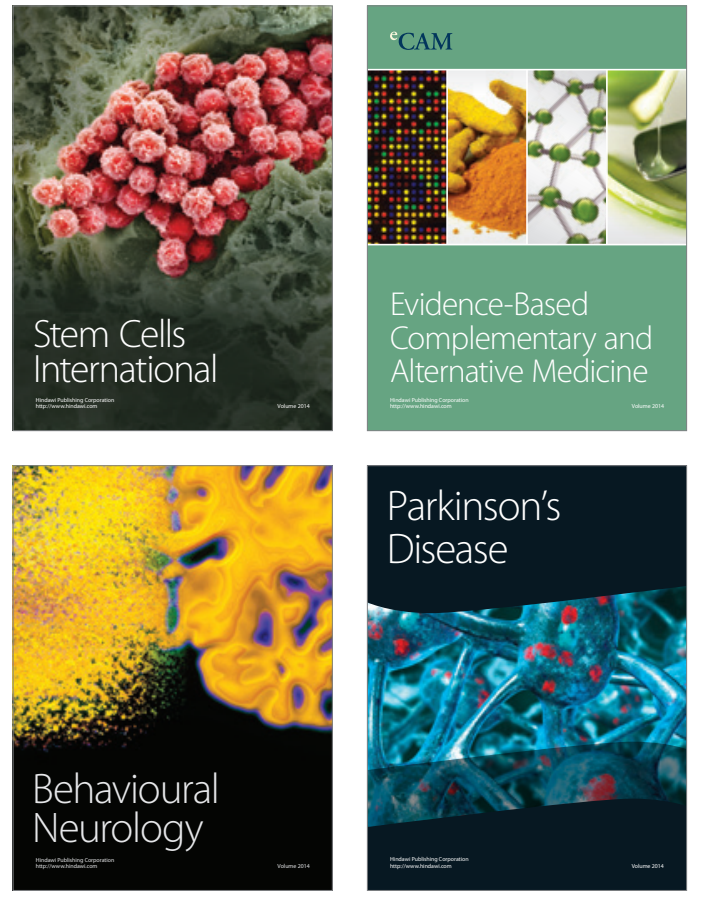
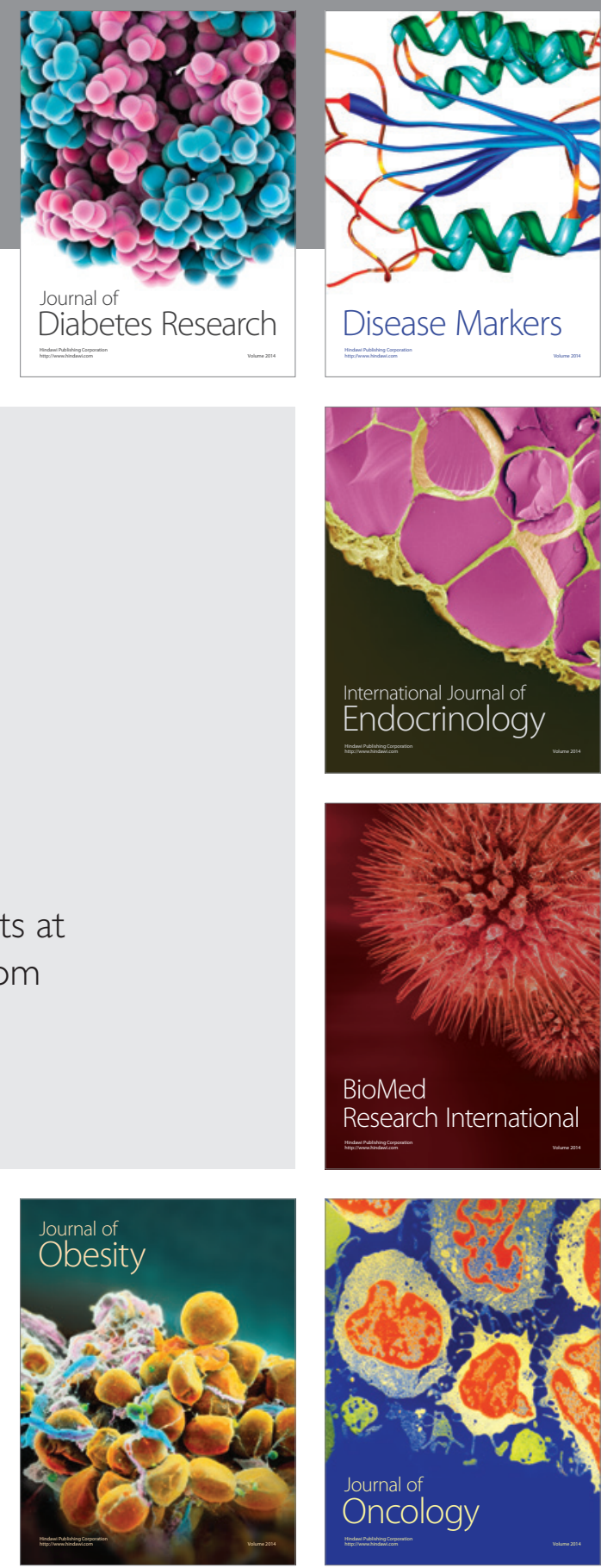

Disease Markers
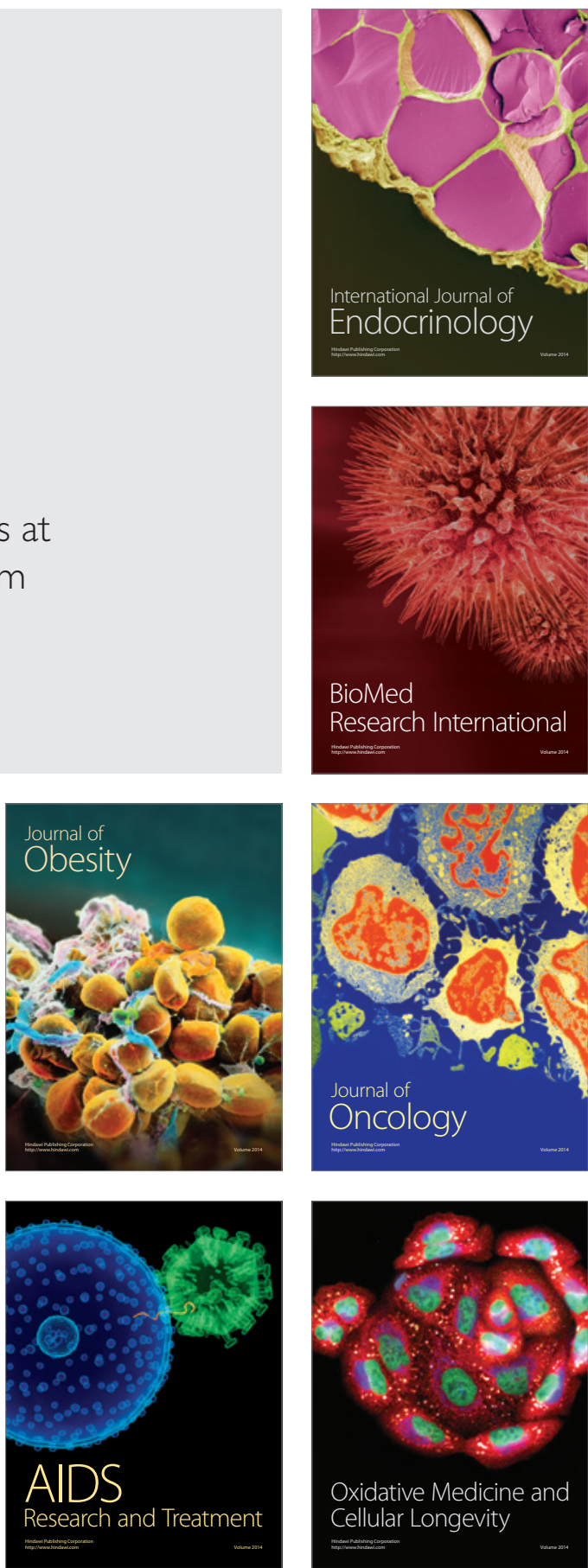